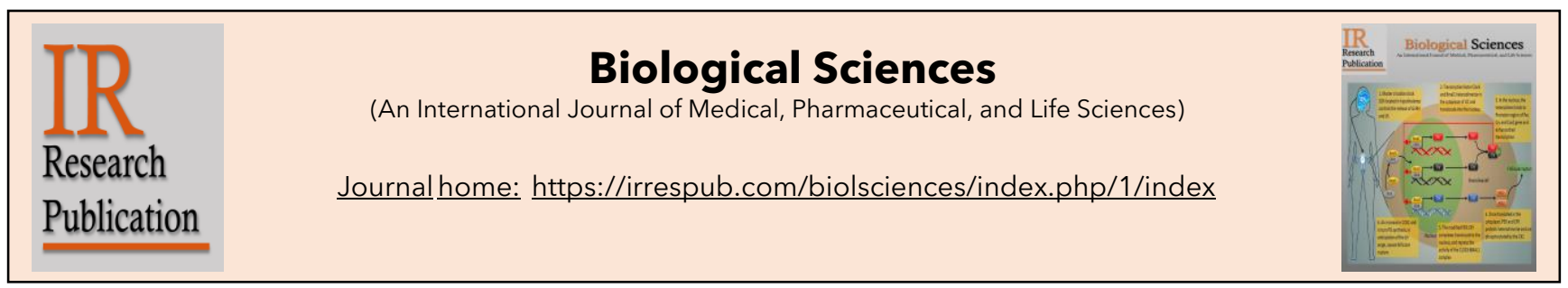

\title{
Amyotrophic lateral sclerosis (ALS) is a complicated challenge that needs distinct evaluation
}

\author{
Manisha Mishra ${ }^{1}$, Disha Sharma ${ }^{1 \star}, \mathrm{Km}$. Shanno ${ }^{2}$, Sudhanshu Mishra ${ }^{1}$ \\ ${ }^{1}$ Department of Pharmacy, DR. M.C. Saxena College of Pharmacy, Lucknow, Uttar Pradesh, India \\ 2Department of Pharmacy, Sri Balaji College of Pharmacy, Jaipur, Rajasthan, India
}

ARTICLE HISTORY

Received: 27-04-2021

Revised: 02-05-2021

Accepted: 02-05-2021

Online: 04-05-2021

KEYWORDS

Amyotrophic lateral sclerosis Neurodegenerative disease Motor Neuron Disease Multidisciplinary care

Sporadic ALS

Familial ALS

\section{ABSTRACT}

The Amyotrophic Lateral Sclerosis (ALS) is a neurodegenerative disease that affects motor neuron and decreases muscle functionality. As it is a cumulative disease, it will eventually head to paralysis and unavoidably death. ALS often has a principal onset, but subsequently spreads to various body regions, where failure of respiratory muscles typically limits survival to 2-5 years after disease onset. The etiology of the ALS is still unclear. However, superoxide dismutase 1 mutations have been known to be the most frequent cause of Familial ALS (FALS). However, despite the low frequency, ALS is not especially infrequent. In up to $50 \%$ of cases, there are extra-motor manifestations such as changes in behaviour, executive dysfunction, and language problems. There is no cure for ALS up till now and the basis of treatment remains multidisciplinary care, including nutritional and respiratory support, and symptom management. Two medications, riluzole and edaravone, have proved effective for the symptomatic treatment with modest benefits, but there is urge for treatment options which can extend the survival rate of the patient. Further, we have discussed different aspects of ALS, including epidemiology, pathogenesis, clinical features, differential diagnosis, investigations, and treatment and future prospects.

\section{Introduction}

Lateral amyotrophic sclerosis is one of five Motor Neuron Disease (MND) that impair motor neurons. Four additional established MNDs are listed: Primary lateral sclerosis (PLS), progressive muscular atrophy

${ }^{\star}$ Address for correspondence

Department of Pharmacy, DR. M.C Saxena College of Pharmacy, Lucknow, Uttar Pradesh, India.

Email: dishasharma.3003@gmail.com

DOI: http://dx.doi.org/10.55006/biolsciences.2021.1109

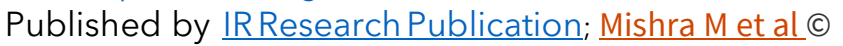

2021 by Biological Sciences is licensed under CC BY 4.0 cc)
(PMA) and progressive bulbar paralysis (PBP). ALS is considered in two forms in which one is Sporadic Amyotrophic Lateral Sclerosis (SALS) and the other is Familial Amyotrophic Lateral Sclerosis (FALS). SALS (90-95\%) is widely renowned, but the segment has no obvious genetically inherited component. The remaining 5-10\% are Familial ALS due to their associated genetic dominant inheritance factor $[1,2,4]$. Nerve cell breakdown in this condition decreases the muscle functionality they have. It is caused by paralysis. Museum fatigue, twitching and cramping, which ultimately will lead to muscle dysfunction, are the most frequent symptoms of both forms of the disease. ALS patients develop dyspnoea, dysarthria, and dysphagia as the disease 
is in the advanced phases. Limb-onset ALS with the combined upper motor neuron (UMN) and lower motor neuron (LMN) signs in the extremities; the bulb initialization ALS, characterized by speech and swallowing difficulties followed by limb weakening in later stages of disease. With 70 percent of patients, Limb-onset ALS is the prevalent form $[3,5,6]$.

The most common symptoms that appear in both types of ALS are muscle weakness, twitching, and cramping, which eventually can lead to the impairment of Muscles. In the most advanced stages, ALS patients will develop symptoms of dyspnea and dysphagia $[2,3,4]$.

The C9ORF72 gene describes the mutations that are occurring in SALS and FALS, contributing up to 6 percent and up to 40 percent respectively, with or without frontotemporal dementia (FTD). The improvement demonstrates a variable percentage of penetration specifically connected to the patient's age. A C9ORF72 gene expansion at molecular level for mRNA transcription decreases the pool of RNA binding proteins and eventually deregulates the metabolism of RNA. Other genes identified in studies of ALS genomes, including neurofilament, peripherin, endothelial vascular (EEDF) growth factor, angiogenin, Motor-Neuron Survival (MNS) and hemochromatosis (HFE).

\section{Epidemiology}

The occurrence of SALS is 2 per 100,000 people per year and nearly 5 per 100,000 . The median setup age is around 60 years. Median survival ranges from 3 to 5 years from onset of symptoms, spanning from 55 to 65 years with a median age of 64 years. The occurrence of SALS before 30 years of age, considered as juvenile sporadic onset and is identified only in 5\% of cases. The life-time occurrence of SALS by 70 years of age was specifically measured to be 1 in 400 . Bulbar onset accounts for $25 \%$ of cases, with an initial trunk or respiratory consequence in the final $5 \%$. Relevant explanation is that several researches have revealed that a significant excess of males is impacted by SALS relative to females, with a male: female ratio of about 2:1 The reason could be attributed to defensive hormones in females. Bulbar onset is more frequent in women and older age groups and is marked by dysarthria and dysphagia. Paralysis is a gradual mechanism that leads to death within 2-3 years for bulbar onset cases and within 3-5 years for limb onset cases $[23,24]$. A precise patient diagnosis is difficult, even for an experienced clinician, because there is a wide variety of motor neuron diseases, such as FALS/SALS, spinal muscular atrophy (SMA), hereditary spastic paraplegia (HSP), primary lateral sclerosis (muscular atrophy (SBMA), or Kennedy disease, that share common and heterogeneous symptoms, such as weakness, spastic paralysis, or both, reflecting a functional loss of upper and/or lower motor neurons.

\section{Pathogenesis}

Intra-neuronal protein aggregates, including TDP43, FUS and SOD1, are well known in pathological trials in patients with ALS and animal disease models. It is hypothesised that aggregates interrupt normal protein homeostasis and cause cellular tension. Aggregates can sequester RNA and other proteins that are important for normal cell function. Physical effects of aggregates can result in impaired axonal transport. Degradation of proteins, which can contribute to energetic depletion of motor neurons, can also affect the turnover of unfolded proteins (Figure 1) $[1,4,5]$.

Various pathological pathways have been involved in different stages of the ALS neurodegenerative process, including mitochondrial dysfunction, oxidative disruption, excitotoxicity, inflammation, and intracellular and nucleocytoplasmic transport defects. Further to the severity of the condition, ALS is not only a neuron dysfunction, it also includes, and probably also needs, other forms of cells, including astrocytes, microglia, macrophages, and potentially oligodendrocytes. [2] The disorder has a focal onset site that progresses through neuroanatomical pathways, likely indicating a prion-like propagation to vulnerable tissues.

There are numerous genetic modifications and potentially environmental risk factors that may contribute to conflicting clinical phenotypes of upper motor neuron disease, lower motor neuron disease, and also FTD, which are likely to cause ALS by both common and distinct pathological mechanisms. Indeed, it has been suspected that several clinical trials in ALS have failed, at least in part, due to possible discrepancies in underlying pathophysiological pathways involving ALS patients $[28,29]$.

\section{Treatment}

The primary goal of ALS treatment is the inhibition of disease progression, although an important secondary consideration is the treatment of damage already done [12]. There is no cure for amyotrophic lateral sclerosis (ALS) but its management revolves around symptomatic and palliative care. Early prognosis offers the best recognition for a prolong, well-being while living with the ailment. Various treatments are used to alleviate symptoms but there are only two pharmacologic agents indicated for the supervision of ALS. Riluzole in the year 1996 had 


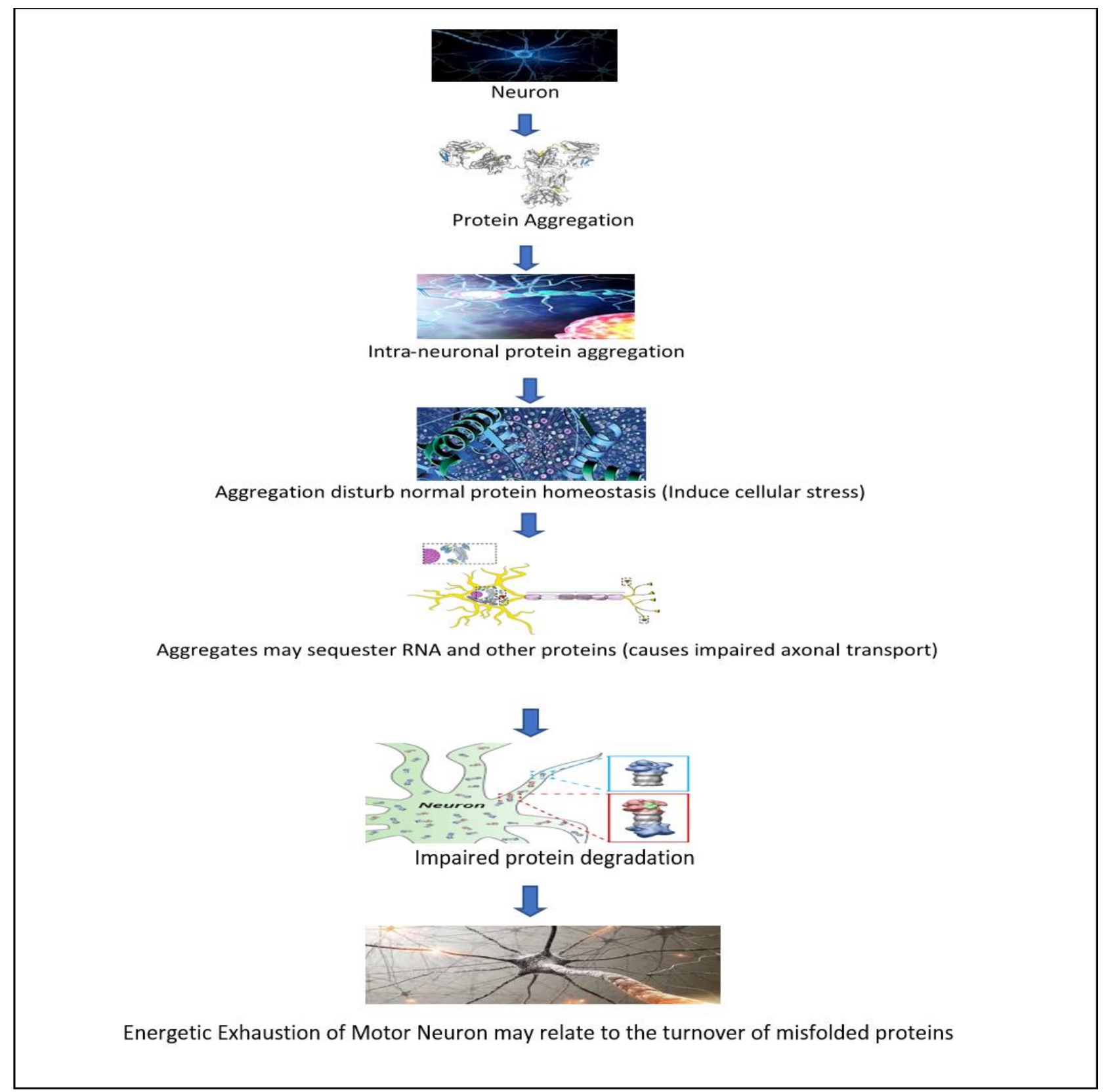

Figure 1. Pathogenesis of amyotrophic lateral sclerosis

been the anchor for disease-modifying therapy, but in May 2017, a new agent Edaravone is approved by the FDA $[11,14,16]$. Various organizations are dedicated to work for multidisciplinary care by imparting aid for people with ALS-support, ALS Research and clinical trial, therapeutic progression for irregular and congenital forms of ALS. There are some other organizations also that support patients with rare disease including ALS (Table 1).

National Organization for Rare Disease (NORD) brings out Patient Assistance Programs that help the patients to obtain life-saving or life-sustaining medication. It administers medication assistance programs for certain rare-disease drugs as well as research grants and fellowships. Other committed organization is Organization for Rare Disease India
(ORDI) that helps to Facilitate \& encourage Clinical Trials, Research \& Orphan drug development activities also provides help in diagnoses and treatment of rare diseases. Also, there is FDA has a long-standing commitment to working with researchers and drug companies and the patient community to facilitate the development and approval of new drug to treat different diseases including rare. WHO (World Health Organization) also provides care and support to the patients that leads the way in research, care to ALS Patients and provides comprehensive patient services and support to the ALS community [8].

\section{Management}

Emphasis has been made on treatments and 
Table 1. List of Organizations that are supporting ALS Patients.

\begin{tabular}{|l|l|}
\hline Organization & Support provided for ALS patient \\
\hline ALS Association & $\begin{array}{l}\text { Association provides services like assistive technology for communication, telemedicine etc for } \\
\text { patient suffering from ALS }\end{array}$ \\
\hline MDA (Muscular Dystrophy ALS) & $\begin{array}{l}\text { Helps to supports muscular dystrophy patients and ALS Patients providing Inpatient care for } \\
\text { diagnostic evaluation, physical and occupational therapy evaluations, diagnostic procedures } \\
\text { including laboratory tests, electrodiagnostic tests and muscle biopsy evaluation for the patients. }\end{array}$ \\
\hline $\begin{array}{l}\text { NEALS (Northeast Amyotrophic } \\
\text { Lateral Sclerosis Consortium) }\end{array}$ & $\begin{array}{l}\text { Support research and clinical trials for ALS Patients that helps in diagnosis of both sporadic and } \\
\text { familial types of ALS, also provides diagnosis and cure to the patients suffering from ALS. }\end{array}$ \\
\hline $\begin{array}{l}\text { CReATe (Clinical Research in } \\
\text { ALS and Related Disorders for } \\
\text { Therapeutic Development) }\end{array}$ & $\begin{array}{l}\text { Advance therapeutic development for sporadic and familial forms of ALS. Promotes } \\
\text { collaborative research for ALS, identify the biomarkers for ALS patients. }\end{array}$ \\
\hline Les Turner ALS Foundation & $\begin{array}{l}\text { Provides best quality of care to the patients and the most promising therapies to the patient } \\
\text { with ALS. }\end{array}$ \\
\hline
\end{tabular}

Table 2. List of drugs that are used for the treatment of ALS patients with its mode of action and clinical uses.

\begin{tabular}{|l|l|l|}
\hline Pharmacological Agents & Mode of action & Clinical Uses \\
\hline Riluzole & $\begin{array}{l}\text { It possesses the inhibitor effect on } \\
\text { release of glutamate }\end{array}$ & Leads to limit down the damage of the motor neuron \\
\hline Edaravone & Free radical scavenger & Prevents oxidative stress \\
\hline
\end{tabular}

interventions that prolong survival. While there are no medications that reverse the progressive loss of neurons, importance has been given to management strategies that optimize the quality of life and help maintain the patient's self-reliance, Multidisciplinary Management, Nutritional Management, Respiratory management, Dietary supplementation may help in the management of ALS patient by - neurologists, physiotherapist, Logopedics, Biotherapist, Gasping therapist, Public servants, dietitians, and nursing care managers for managing patients with ALS to enhance health care delivery, prolong survival, and the quality of life $[24,25,27]$. Riluzole is thought to mitigate damage to motor neurons through inhibiting the liberation of glutamate, whereas Edaravone is thought to function as a neuroprotective agent that inhibits oxidative stress damage as a free radical scavenger Owing to the scarcity of treatment choices, it is important to consider the consequences of using these two agents for improving therapy and wellbeing in patients with ALS (Table 2) $[1,5,6]$.

\section{Conclusion}

The study of ALS gives various information from epidemiology to molecular mechanism and treatment of disease. Amyotrophic lateral sclerosis is a cataclysmic neurodegenerative condition that typically begins with focal muscle weakness and eventually progresses to death from respiratory failure $[21,22$,$] . Although there is no cure for ALS,$ treatment can improve both the quality and extent of life. Two medications, Riluzole and edaravone, have proved effective for the medication of ALS, but more treatments are needed. Current treatment strategies are otherwise largely palliative, aiming to provide better well-being and prolonged survival, with assisted ventilation that is being the most powerful approach. To transform toward more efficient therapies, clinical trials must be conducted with flawless stratification based on rapid/slow advancement and consequent retrogression. Pharmaco-metabolomics allows the identification of biomarkers that are acclimated for a given drug $[19,20]$ The gradual translational stride suggests that current research models are not ideal to study such a sophisticated disease, therefore it needs to be re-framed. $[17,18]$ The breakthrough will require greater intuition into human genes and natural science involved in ALS susceptibility, as well as a deeper understanding of disease constitution at the cytological and molecular levels. Direct focus towards improved assessment technologies and innovative approaches has been made for Improving the outcomes of human diseases $[9,10]$. Future conduct regarding the therapeutic potential of stem cells in ALS, that focus on mesenchymal stem cells, with their high proliferation activity. The immunomodulation activity, multi-differentiation potential, and the capacity to secrete neuroprotective factors, adult mesenchymal stem cells represent promising possibilities for clinical translation. However, there are some technical 
hurdles such as optimal dose, differentiation state, route of administration, and the underlying potential therapeutic mechanisms that are still needed to be evaluated $[3,8,9,11]$.

\section{Acknowledgements}

Authors are grateful to Department of Pharmacy, Dr. M.C. Saxena Group of Colleges, Lucknow and Sri Balaji College of pharmacy, Jaipur for their undue support regarding this.

\section{Conflict of interest}

Authors do not have any conflict of interest.

\section{References}

1. Masrori, P., \& Van Damme, P. 2020. Amyotrophic lateral sclerosis:a clinical review. European Journal of Neurology.

2. Morgan, S., \&Orrell, R. W.2010. Pathogenesis of amyotrophic lateral sclerosis. British Medical Bulletin,119(1), 87-98.

3. Calvo, A. C., Manzano, R., Mendonça, D. M. F., Muñoz, M. J., Zaragoza, P., \&Osta, R. 2014. Amyotrophic Lateral Sclerosis: A Focus on Disease Progression. BioMed Research International,2014, 1-12.

4. Oskarsson, B., Gendron, T. F., \& Staff, N. P. 2018. Amyotrophic Lateral Sclerosis: An Update for 2018. Mayo Clinic Proceedings.

5. Stéphane Mathisa., Cyril Goizetc.,AntoineSoulagesa., Jean-Michel Vallate., Gwendal Le Massona. Genetics of amyotrophic lateral sclerosis: A review. Journal of the Neurological Sciences 3992019 217-226.

6. Mitsumoto, H. (1997). Riluzole - What is its Impact in Our Treatment and Understanding of Amyotrophic Lateral Sclerosis? Annals of Pharmacotherapy,31(6), 779-781.

7. Vicencio., Beltrán., Labrador., Manque., Nassif., \&Woehlbier. 2020. Implications of Selective Autophagy Dysfunction for ALS Pathology. Cells, 9(2), 381.

8. Center for disease Control and Prevention: National Amyotrophic Lateral Sclerosis (ALS) Registry: https://www.cdc.gov/als/OrganizationsthatSupp ortPALS.html

9. Clerc, P., Lipnick, S., \& Willett, C. 2019. A look into the future of ALS research. Drug Discovery Today, 21(6), 939-949.

10.Hergesheimer, R., Lanznaster, D., Vourc'h, P., Andres, C., Bakkouche, S., Beltran, S., ... Couratier, P. 2020. Advances in diseasemodifying pharmacotherapies for the treatment of amyotrophic lateral sclerosis. Expert Opinion on Pharmacotherapy, 1-8.
11.Ciervo, Y., Ning, K., Jun, X., Shaw, P. J., \& Mead, R. J. 2017. Advances, challenges and future directions for stem cell therapy in amyotrophic lateral sclerosis. Molecular Neurodegeneration, 12(1).

12.Perry, J., Pratt, \&Getzoff. 2012. Amyotrophic lateral sclerosis: update and new developments. Degenerative Neurological and Neuromuscular Disease, 1.

13.Salameh, J., Brown, R., \& Berry, J. 2015. Amyotrophic Lateral Sclerosis: Review. Seminars in Neurology, 35(04), 469-476.

14.Masrori, P., \& Van Damme, P. 2020. Amyotrophic lateral sclerosis: a clinical review. European Journal of Neurology.

15.Wobst, H. J., Mack, K. L., Brown, D. G., Brandon, N. J., \& Shorter, J. 2020. The clinical trial landscape in amyotrophic lateral sclerosis-Past, present, and future. Medicinal Research Reviews.

16.Kuraszkiewicz, B., Goszczyńska, H., PodsiadłyMarczykowska, T., Piotrkiewicz, M., Andersen, P., Gromicho, M., ... de Carvalho, M. (2020). Potential Preventive Strategies for Amyotrophic Lateral Sclerosis. Frontiers in Neuroscience, 14.

17.Mejzini, R., Flynn, L. L., Pitout, I. L., Fletcher, S., Wilton, S. D., \&Akkari, P. A. 2019. ALS Genetics, Mechanisms, and Therapeutics: Where Are We Now? Frontiers in Neuroscience, 13.

18.Longinetti, E., \& Fang, F. 2019. Epidemiology of amyotrophic lateral sclerosis. Current Opinion in Neurology, 32(5), 771-776.

19.Volk, A. E., Weishaupt, J. H., Andersen, P. M., Ludolph, A. C., \&Kubisch, C. 2018. Current knowledge and recent insights into the genetic basis of amyotrophic lateral sclerosis. MedizinischeGenetik, 30(2), 252-258.

20.Grad, L. I., Rouleau, G. A., Ravits, J., \& Cashman, N. R.2016. Clinical Spectrum of Amyotrophic Lateral Sclerosis (ALS). Cold Spring Harbor Perspectives in Medicine, 7(8), a024117.

21.Riva, N., Agosta, F., Lunetta, C., Filippi, M., \&Quattrini, A. 2016. Recent advances in amyotrophic lateral sclerosis. Journal of Neurology, 263(6), 1241-1254.

22.Gordon, P. H. 2011. Amyotrophic Lateral Sclerosis. CNS Drugs, 25(1), 1-15.

23.Borasio, G. D., \& Miller, R. G. 2001. Clinical Characteristics and Management of ALS. Seminars in Neurology, 21(02), 155-166.

24.Martin, S., Al Khleifat, A., \& Al-Chalabi, A. 2017. What causes amyotrophic lateral sclerosis? F1000Research, 6, 371.

25.Bensimon, G., Lacomblez, L., Delumeau, J. C., Bejuit, R., Truffinet, P., \&Meininger, V. 2002. A study of riluzole in the treatment of advanced stage or elderly patients with amyotrophic lateral sclerosis. Journal of Neurology, 249(5), 609-615.

26.Ghasemi, M., \& Brown, R. H. 2017. Genetics of Amyotrophic Lateral Sclerosis. Cold Spring Harbor Perspectives in Medicine, 8(5), a024125. 
27.Logroscino, G., Traynor, B. J., Hardiman, O., Chio', A., Couratier, P., ... Mitchell, J. D. 2008. Descriptive epidemiology of amyotrophic lateral sclerosis: new evidence and unsolved issues. Journal of Neurology, Neurosurgery \& Psychiatry, 79(1), 6-11.

28.Van Damme, P., Robberecht, W., \& Van Den Bosch, L. 2017. Modelling amyotrophic lateral sclerosis: progress and possibilities. Disease Models \& Mechanisms, 10(5), 537-549.

29.Mangelsdorf, I., Walach, H., \& Mutter, J. 2017. Healing of Amyotrophic Lateral Sclerosis: A Case Report. Complementary Medicine Research, 24(3), 175-181.

30.Hogden, A., Foley, G., Henderson, R., James, N., \& Aoun, S. 2017. Amyotrophic lateral sclerosis: improving care with a multidisciplinary approach. Journal of Multidisciplinary Healthcare, Volume 10, 205-215. 